\title{
Chiropractic spinal manipulative therapy for cervicogenic headache: a single-blinded, placebo, randomized controlled trial
}

\author{
Aleksander Chaibi ${ }^{1,2^{*}}$ (D, Heidi Knackstedt ${ }^{3}$, Peter J. Tuchin ${ }^{4}$ and Michael Bjørn Russell ${ }^{1,2}$
}

\begin{abstract}
Objective: Cervicogenic headache is a disabling headache where pharmacological management have limited effect. Thus, non-pharmacological management is warranted. Our objective was therefore to investigate the efficacy of chiropractic spinal manipulative therapy versus placebo (sham manipulation) and control (continued usual but non-manual management) for cervicogenic headache in a prospective 3-armed single-blinded, placebo, randomized controlled trial of 17 months' duration.
\end{abstract}

Results: Nineteen participants were equally randomized into the three groups, and 12 participants completed the randomized controlled trial. Headache frequency improved at all time points in the chiropractic spinal manipulative therapy and the placebo group. Headache index improved in the chiropractic spinal manipulative therapy group at all time points, while it improved at 6 and 12 months' follow-up in the placebo group. The control group remained unchanged during the whole study period. Adverse events were few, mild and transient. Blinding was concealed throughout the RCT. Thus, our results suggest that manual-therapy might be a safe treatment option for participants with cervicogenic headache, but data need to be confirmed in a randomized controlled trial with sufficient sample size and statistical power.

Trial registration ClinicalTrials.gov identifier: NCT01687881, 11 September 2012

Keywords: Randomized controlled trial, Cervicogenic headache, Headache, Chiropractic, Spinal manipulative therapy, RCT

\section{Introduction}

Cervicogenic headache $(\mathrm{CEH})$ is a secondary headache characterized by unilateral headache, and symptoms and signs of neck involvement $[1,2]$. It is often worsened by neck movement, sustained awkward head position or external pressure over the upper cervical or occipital region on the symptomatic side $[1,2]$. The prevalence of $\mathrm{CEH}$ varies from 1.0 to $4.6 \%$ in the general population depending on the diagnostic criteria $[1,3-6]$. The efficacy of pharmacological management for $\mathrm{CEH}$ is poor and medication overuse is frequent [7]. Due to insufficient pharmacological treatment

\footnotetext{
*Correspondence: aleksander.chaibi@medisin.uio.no

${ }^{1}$ Head and Neck Research Group, Research Centre, Akershus University

Hospital, 1478 Lørenskog, Norway

Full list of author information is available at the end of the article
}

strategies, spinal manipulative therapy (SMT) has been recommended as a treatment option, despite the methodological shortcomings found in previous manual-therapy clinical trials $[8,9]$; especially threearmed studies, including an active, a placebo, and a control group, have not previously been conducted, although this is recommended as the gold standard in RCTs.

The primary objective was to investigate the efficacy of chiropractic spinal manipulative therapy (CSMT) versus placebo (sham manipulation) and CSMT versus control (continued usual management but no manual-therapy were allowed during the trial period) in participants with $\mathrm{CEH}$. 


\section{Main text \\ Methods \\ Design}

This study was a prospective triple-armed, placebo RCT of 17 months' duration with single blinded treatment and blinded outcome measures. The trial consisted of 1 month baseline, 3 months' treatment with a total of 12 intervention sessions, and follow-up analysis at end of intervention and 3, 6, 12 months'. Participants were block randomized into: (a) CSMT, (b) placebo (sham manipulation), or (c) control (continued usual management but no manual-therapy were allowed during the trial period). The study design conforms the International Headache Society (IHS) and CONSORT [10-13]. The full trial protocol has been published previously and contains explicit details regarding the methodology [14].

\section{Participants}

Participants were recruited from September to October 2012 through the Akershus University Hospital and Innlandet Hospital Trust, Norway.

Eligible participants were between 18 and 70 years of age, diagnosed with a CEH by a neurologist, including at least three major criteria of the CHISG but not including occipital nerve blockage [1]. Exclusion criteria are described in details in the available protocol [14].

Eligible participants were invited to an interview and physical assessment by a chiropractor (AC) including meticulous investigation of the spinal column. Participants, who were randomized to CSMT or placebo, had a full spine radiographic examination prior to intervention commenced.

\section{Intervention}

The CSMT group received SMT using the Gonstead method, directed to spinal biomechanical dysfunction as diagnosed by standard chiropractic tests [15].

The placebo group received sham manipulation at the lateral edge of the scapula and/or the gluteal region [16].

The control group continued their usual pharmacological management without receiving manual intervention.

The interventions are described in details in the available trial protocol [14].

\section{Blinding}

After each treatment session, participants completed a de-blinding questionnaire, see protocol for details [16].

\section{Outcome measures}

Headache characteristics were recorded in the chiropractic in-depth interview followed by 1 month of validated diagnostic headache diary for baseline recordings [17], which was returned on a monthly basis [14].
The primary end-point was $25 \%$ reduction in number of headaches days per month (30 days/month) from baseline to end of intervention and 3, 6 and 12 months follow-up as compared to the placebo group and the control group respectively. Secondary end-points included a $25 \%$ improvement in headache duration, headache intensity and headache index (HI). HI was calculated as mean days with headache (30 days) $\times$ mean headache duration (hours per day) $\times$ mean intensity $(0-10 \mathrm{NRS})$. Endpoints were based on IHS clinical guidelines $[10,11]$.

All adverse events (AEs) were recorded after each consultation in accordance with the CONSORT recommendations and IHS Task Force on AEs in migraine trials [12, 13].

\section{Power calculation}

The power was based on a recent group comparison study of topiramate [18]. A sample size of 20 patients was required in each group to detect a statistical significant average difference in reduction of $25 \%$ with $80 \%$ power, see protocol for details [14].

\section{Statistical analysis}

Data were recorded in MS Excel 2007. All analysis was performed by a blinded investigator using sealed serial number for each participant. Results for primary and secondary end-points are presented individually for each participant while mean change for each group are presented in figures. Mean $\mathrm{HI}$ and $95 \%$ confidence interval (CI) was calculated for baseline characteristics using SPSS v22.

\section{Ethics and data security}

All methods were carried out in accordance with the approved guidelines and regulations. Ethical details are available in the trial protocol [14].

\section{Results}

Ninety-five participants with CEH diagnosed by a neurologist at Akershus University Hospital or Innlandet Hospital Trust, Norway were contacted. Twenty-one did not reply to the 1st or 2nd invitation letter. Among the 74 participants screened by telephone, 27 participants did not meet the inclusion criteria, i.e., nine (two men and seven women) had received CSMT within the last 12 months, four (one man and three women) had spinal radiculopathy, one woman had a dens fracture, one woman had a vertebral artery insufficiency, one woman had a cervical spine tumour, five (two men and three women) had headache remission, three (one man and two women) suffered depression, one woman had a brain tumour, one woman had insufficient Norwegian language skills, and one woman was pregnant. 
Among the 47 eligible participants, 28 refrained to participate, i.e., due to time concerns ( 1 man and 11 women), physicians advised against participation (one man and two women), no faith in the treatment (one man and one woman), and unknown reason(s) (eight men and three women).

A total of 19 participants (5 men and 14 women) were randomized (Additional file 1). Three participants dropped out and four participants were excluded after randomization. Reasons for dropping out included, refrained to keep a headache diary (one woman), refrained to stop physiotherapy (one woman), and inclusion in an obesity rehabilitation program (one woman). Reasons for exclusion included, headache diaries not returned (two women), depression (one woman) and inflammatory disease (one man). Four participants were allocated to each of the three interventions. Baseline characteristic across the three groups were similar (Table 1).

\section{Outcome measures}

Individual changes in headache frequency, duration, intensity and $\mathrm{HI}$ from baseline to post-treatment and 3, 6 and 12 months follow-up are presented in Table 2.

Mean change in primary and secondary end-points for each group are presented in Fig. 1a-d.

The main effect of the treatment was on headache frequency in both the CSMT and the placebo group, an effect that was maintained at follow-up (Fig. 1a).

Headache index improved in the CSMT group at all time points while it improved at 6 and 12 months followup in the placebo group.
The control group remained unchanged during the whole study period (Fig. 1a-d).

\section{Blinding}

The four participants who received CSMT believed they received it with a mean 9.1 certainty, whereas the four participants who received placebo believed they received active CSMT with a mean 6.4 certainty.

\section{Adverse effects}

Mild transient AEs were local tenderness and tiredness on the treatment day in both the CSMT and the placebo group. No severe or serious adverse events were reported in the study.

\section{Discussion}

To our knowledge, this is the first triple-armed chiropractic manual-therapy RCT to include a placebo and a control group for participants with $\mathrm{CEH}$. The study is also the first to successfully maintain blinding throughout a full length intervention period. Our main results demonstrate reduction in headache frequency and $\mathrm{HI}$ in the CSMT and the placebo group, an effect that lasted at follow-up, while the control group was unchanged throughout the RCT.

\section{Methodological considerations}

As compared to previous manual-therapy RCTs, our participants were selected based on more rigorous criteria for $\mathrm{CEH}$ and the diagnosis was confirmed by a neurologist experienced in headache disorders [9]. The exclusion criteria i.e., depression, chiropractic treatment within the

Table 1 Baseline demographic and clinical characteristics

\begin{tabular}{|c|c|c|c|}
\hline & Chiropractic spinal manipulative therapy & Sham manipulation (placebo) & Control group \\
\hline Number of participants & 4 & 4 & 4 \\
\hline Males & 1 & 1 & 2 \\
\hline Females & 3 & 3 & 2 \\
\hline Age \pm SD (range) & $36.0 \pm 12.8(26-54)$ & $49.8 \pm 12.3(32-58)$ & $48.0 \pm 9.8(39-61)$ \\
\hline Duration (years with headache \pm SD) & $7.3 \pm 3.3$ & $8.5 \pm 1.3$ & $13.8 \pm 10.4$ \\
\hline Headache frequency (mean 30 days/month) & $20.5 \pm 9.9$ & $25.5 \pm 5.2$ & $19.3 \pm 7.9$ \\
\hline Headache duration (mean hours/day) & $19.5 \pm 3.8$ & $19.5 \pm 7.7$ & $19.3 \pm 5.1$ \\
\hline Headache intensity (mean 0-10 VAS) & $6.0 \pm 2.3$ & $6.0 \pm 0.7$ & $7.0 \pm 2.0$ \\
\hline Co-morbid migraine & $0 / 4$ & $2 / 4$ & $0 / 4$ \\
\hline \multicolumn{4}{|l|}{ Primary end-point (1 month baseline) } \\
\hline Headache frequency (mean 30 days/month) & $20.0 \pm 12.0$ & $22.8 \pm 12.3$ & $19.0 \pm 8.4$ \\
\hline \multicolumn{4}{|l|}{ Secondary end-points (1 month baseline) } \\
\hline Headache duration (mean hours/day) & $9.6 \pm 5.2$ & $10.9 \pm 3.3$ & $9.9 \pm 6.9$ \\
\hline Headache intensity (mean 0-10 VAS) & $6.6 \pm 2.5$ & $4.3 \pm 1.7$ & $6.3 \pm 1.6$ \\
\hline $\begin{array}{l}\text { Headache index }(\mathrm{HI}) \\
\text { (mean frequency } \times \text { duration } \times \text { intensity) }\end{array}$ & $\begin{array}{l}956 \\
95 \% \text { Cl } 438 \text { to } 1475\end{array}$ & $\begin{array}{l}1141 \\
95 \% \text { Cl } 283 \text { to } 1999\end{array}$ & $\begin{array}{l}1635 \\
95 \% \mathrm{Cl}-37 \text { to } 3307\end{array}$ \\
\hline
\end{tabular}




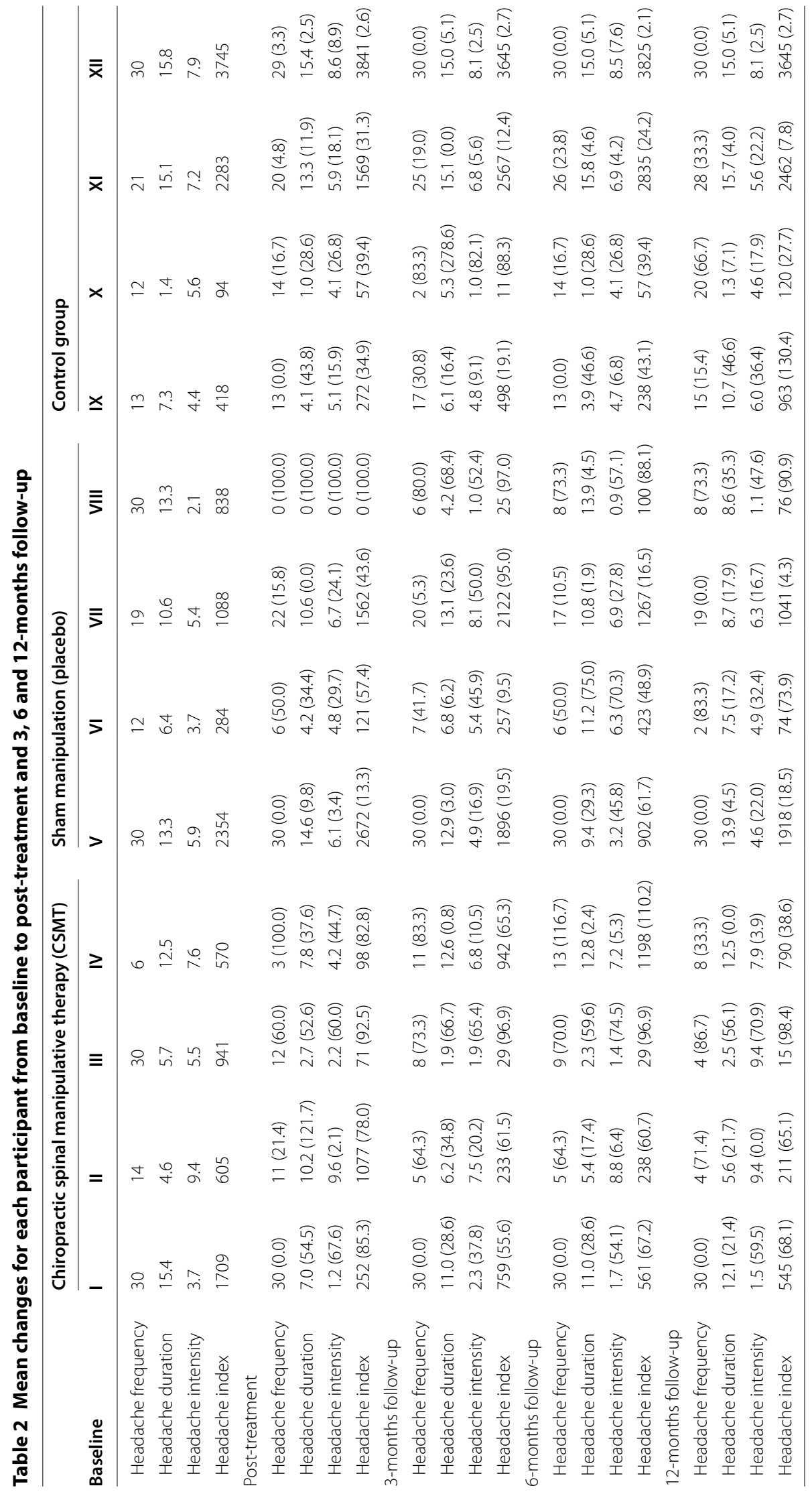



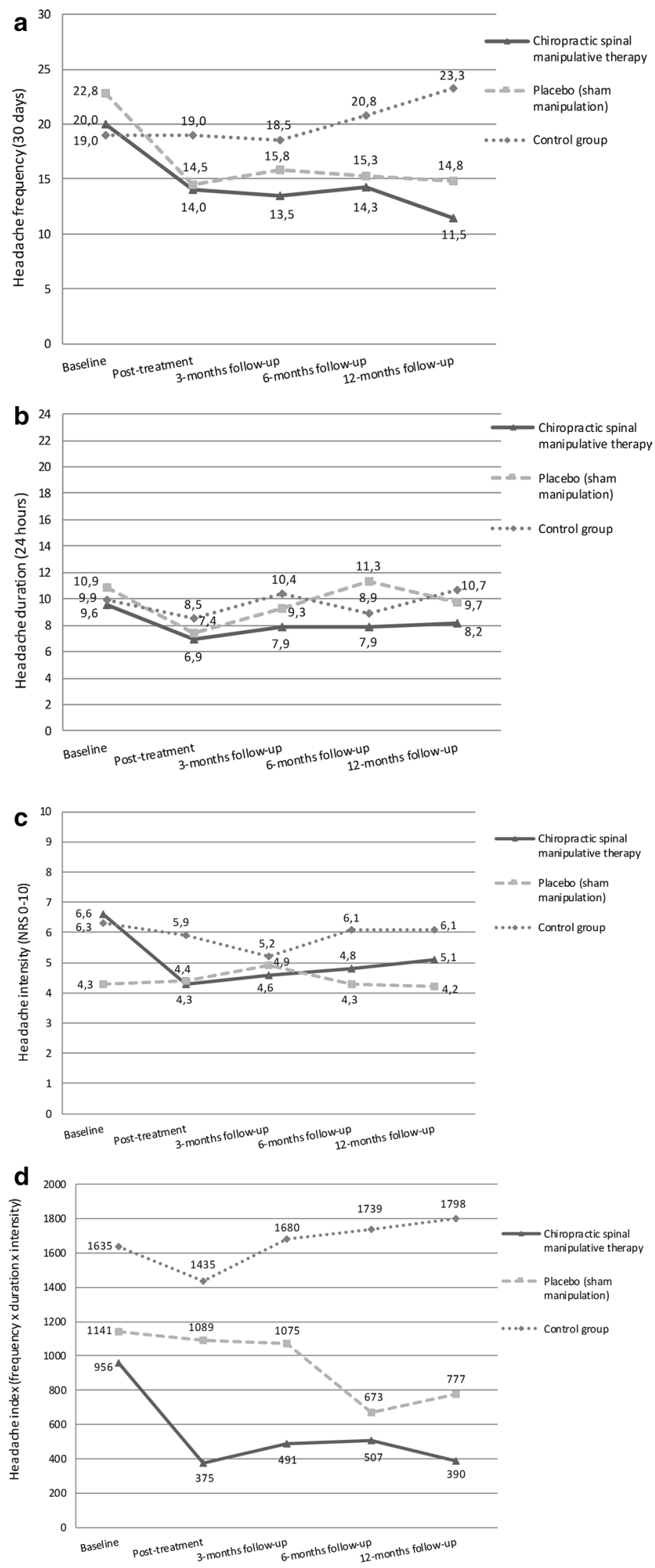

Fig. 1 a-d Mean changes from baseline to follow-up for primary and secondary end-points 
previous 12 months and pregnancy, excluded 36\% of the participants. Furthermore, rigorous RCT rules limiting participants to omit from any manual therapies throughout the 12 months' follow-up period was necessary to obtain a homogenous sample population, avoid type-II errors, and enable a successful blinding in the placebo group.

Similar to other studies of $\mathrm{CEH}$, and considering that the prevalence of $\mathrm{CEH}$ is $0.17 \%$ in the general Norwegian population [7], we experienced severe challenges in recruiting and maintaining participants in the trial. Thus, we can only present descriptive data. Although a cross-over design would have strengthen the power, several limitations exists with this design, i.e., (a) longer intervention period extended by a wash-out period; (b) the ethical concerns regarding switching from a successful treatment to placebo; (c) reports of AEs could possible unmask the blinding. It was also surprising that $38 \%$ declined to participate, primarily due to time concerns which might indicate that their $\mathrm{CEH}$ might be less severe or lack of enthusiasm due to previous therapy failures [19].

The strength of our RCT includes diagnosis and intervention by a single experienced chiropractor which contribute to a strong internal validity. Prospective headache diaries give near exact measurements. The HI has despite the lack of consensus been recommended as a measurement outcome to give an indication of the total level of suffering [11].

\section{Results discussion}

The results in this trial are similar to previous results reported in reviews on SMT for CEH $[9,20]$. A Danish RCT found headache intensity to reduce by 36 and $22 \%$ in the SMT group at post treatment and 1 week followup respectively as compared to the soft tissue treatment group [21]. An Australian RCT with high methodological quality reported $71 \%$ of the participants having $>50 \%$ reduction in headache frequency while $33 \%$ reported a $100 \%$ improvement in the SMT group [22]. Two American RCTs reported a mean reduction of 43, 29 and 40\% reduction in headache intensity at 4, 12 and 24 weeks follow-up respectively in the SMT group, while mean headache frequency similarly reduced by 49,34 and $52 \%$ respectively $[23,24]$.

The placebo effect is known to be high in headache RCTs and assumed similar high for non-pharmacological clinical trials [25-27]. The placebo effect for headache frequency was high in our RCT, while HI improved first at 6 months follow-up in the placebo group. One should therefore not disregard the fact that the effect shown in our RCT could be a placebo effect.
A confounding factor for which no improvement in days with headache was omitted for one participant in the CSMT group, i.e., participant one; and two participants in the placebo group, i.e., participant five and seven according to their diaries, was the fact that they probably suffered medication overuse headache [2].

It is not uncommon that participants receiving placebo intervention report similar AEs as seen in the active intervention, likely produced by expectations $[28,29]$. AEs following placebo administration in pharmacological clinical trials for primary headache disorders has been reported as high as $43 \%$ [30], often related to the study information letter, the informed consent and attitude towards participants. Considering our success in concealing the blinding, the latter fact seems unlikely. However, the true nature of the nocebo effects cannot be identified from our three-armed RCT since the control group was included to quantify the placebo effect, and AEs was not monitored in the control group.

Finally, one participant in the placebo group i.e., participant eight, experienced daily headache frequency at baseline and no headache post-treatment, which slightly increased at 3, 6 and 12 months follow-up. This certainly influenced the results in the placebo group, and the complete resolution of the headache at post treatment stay in contrast to the other participants, and one can speculate whether this is a real phenomenon or an issue of noncompliance when returning the headache diary.

Given the limited effect of pharmacological management for $\mathrm{CEH}$, this study adds knowledge to previous observed effects from SMT [9]. Unfortunately, the low sample size limits our conclusions; replications of this trial with a substantial increased sample size which follows the recommended IHS clinical trial guidelines are needed to confirm the results $[10,11]$. Our attempt to blind participants, in order to establish a placebo group in a manual-therapy RCT was our most successful result.

\section{Limitations}

Small sample size enforced us to present descriptive data and limits our conclusions.

\section{Additional file}

Additional file 1. Participants flow diagram.

\section{Abbreviations}

AE: adverse event; $C E H$ : cervicogenic headache; $\mathrm{Cl}$ : confidence interval; CSMT: chiropractic spinal manipulative therapy; HI: headache index; HVLA: high velocity low amplitude; IHS: International Headache Society; $\mathrm{MOH}$ : medication overuse headache; RCT: randomized controlled trial; SMT: spinal manipulative therapy. 


\begin{abstract}
Authors' contributions
MBR had the original idea of the study and planned the overall design. AC prepared the initial draft, conducted the treatments and is the main author of the present manuscript. HK collected data and diagnosed invited participants. AC, PT and MBR were involved in data analysis and interpretation and assisted in preparation of the manuscript. All authors read and approved the final manuscript.
\end{abstract}

\section{Author details}

${ }^{1}$ Head and Neck Research Group, Research Centre, Akershus University Hospital, 1478 Lørenskog, Norway. ${ }^{2}$ Institute of Clinical Medicine, Akershus University Hospital, University of Oslo, 1474 Nordbyhagen, Norway. ${ }^{3}$ Department of Neurology, Innlandet Hospital Trust, 2418 Elverum, Norway. ${ }^{4}$ Department of Chiropractic, Macquarie University, Sydney, NSW 2109, Australia.

\section{Acknowledgements}

Akershus University Hospital and Innlandet Hospital Trust, Norway, kindly provided research facilities. Innlandet Hospital Trust, Department of Radiology, Elverum and Chiropractor Clinic 1, Oslo, Norway performed x-rays assessment. We would also like to acknowledge prof. Jūratė Šaltytė Benth for her statistical inputs to the study.

\section{Competing interests}

The authors declare that they have no competing interests.

\section{Availability of data and materials}

No additional data are available for sharing.

\section{Consent for publication}

Not applicable.

\section{Ethical approval}

The Norwegian Regional Committee for Medical Research Ethics approved the project (ID of the approval: 2010/1639/REK) and the Norwegian Social Science Data Services (ID no. 11-77, Akershus University Hospital) approved the project. Oral and written consent was obtained from all participants.

\section{Funding}

The study has received funding from Extrastiftelsen (Grant Number: 2829002), the Norwegian Chiropractic Association (Grant Number: 2829001), Akershus University Hospital (Grant Number: N/A) and University of Oslo in Norway (Grant Number: N/A). The funding sources were non-commercial, and the study was designed and conducted by the authors without any influence by the funding sources

\section{Publisher's Note}

Springer Nature remains neutral with regard to jurisdictional claims in published maps and institutional affiliations.

Received: 29 May 2017 Accepted: 21 July 2017

Published online: 24 July 2017

\section{References}

1. Sjaastad O, Fredriksen TA, Pfaffenrath V. Cervicogenic headache: diagnostic criteria. The cervicogenic headache international study group. Headache. 1998;38:442-5.

2. ICHD: Headache Classification Subcommittee of the International Headache Society. The international classification of headache disorders, 3rd edition (beta version). Cephalalgia. 2013;33:629-808

3. Nilsson $\mathrm{N}$. The prevalence of cervicogenic headache in a random population sample of 20-59 year olds. Spine (Phila Pa 1976). 1995;20:1884-8.

4. Pareira Monteriro J. Epidemiologic e clinico de uma populacäo urbana. Porto: University of Porto, Cefaleia; 1995.

5. ICHD: Headache Classification Subcommittee of the International Headache Society. The international classification of headache disorders, 2nd edition. Cephalalgia. 2004;24(Suppl 1):9-160.
6. Sjaastad O, Bakketeig LS. Prevalence of cervicogenic headache: Vaga study of headache epidemiology. Acta Neurol Scand. 2008;117:173-80.

7. Knackstedt H, Bansevicius D, Aaseth K, Grande RB, Lundqvist C, Russell MB. Cervicogenic headache in the general population: the Akershus study of chronic headache. Cephalalgia. 2010;30:1468-76.

8. Fernandez-de-las-Penas C, Alonso-Blanco C, San-Roman J, MiangolarraPage JC. Methodological quality of randomized controlled trials of spinal manipulation and mobilization in tension-type headache, migraine, and cervicogenic headache. J Orthop Sports Phys Ther. 2006;36:160-9.

9. Chaibi A, Russell MB. Manual therapies for cervicogenic headache: a systematic review. J Headache Pain. 2012:13:351-9.

10. Tfelt-Hansen P, Block G, Dahlof C, Diener HC, Ferrari MD, Goadsby PJ, Guidetti V, Jones B, Lipton RB, Massiou H, International Headache Society Clinical Trial Subcommittee, et al. Guidelines for controlled trials of drugs in migraine: second edition. Cephalalgia. 2000;20:765-86.

11. Silberstein S, Tfelt-Hansen P, Dodick DW, Limmroth V, Lipton RB, Pascual J, Wang SJ. Task Force of the International Headache Society Clinical Trial Subcommittee. Guidelines for controlled trials of prophylactic treatment of chronic migraine in adults. Cephalalgia. 2008;28:484-95.

12. Tfelt-Hansen P, Bjarnason NH, Dahlof C, Derry S, Loder E, Massiou H. Evaluation and registration of adverse events in clinical drug trials in migraine. Cephalalgia. 2008;28:683-8.

13. Moher D, Hopewell S, Schulz KF, Montori V, Gotzsche PC, Devereaux PJ, Elbourne D, Egger M, Altman DG. CONSORT 2010 explanation and elaboration: updated guidelines for reporting parallel group randomised trials. BMJ. 2010;340:C869.

14. Chaibi A, Benth JS, Tuchin PJ, Russell MB. Chiropractic spinal manipulative therapy for cervicogenic headache: a study protocol of a single-blinded placebo-controlled randomized clinical trial. Springerplus. 2015:4:779.

15. Cooperstein R. Gonstead chiropractic technique (GCT). J Chiropr Med. 2003;2:16-24

16. Chaibi A, Benth JS, Russell MB. Validation of placebo in a manual therapy randomized controlled trial. Sci Rep. 2015:5:11774.

17. Russell MB, Rasmussen BK, Brennum J, Iversen HK, Jensen RA, Olesen J. Presentation of a new instrument: the diagnostic headache diary. Cephalalgia. 1992;12:369-74.

18. Silberstein SD, Neto W, Schmitt J, Jacobs D. Topiramate in migraine prevention: results of a large controlled trial. Arch Neurol. 2004;61:490-5.

19. Aaseth K, Grande RB, Benth JS, Lundqvist C, Russell MB. 3-year follow-up of secondary chronic headaches: the Akershus study of chronic headache. Eur J Pain. 2011;15:186-92

20. Fernandez-de-las-Penas C, Alonso-Blanco C, Cuadrado ML, Pareja JA. Spinal manipulative therapy in the management of cervicogenic headache. Headache. 2005:45:1260-3.

21. Nilsson N. A randomized controlled trial of the effect of spinal manipulation in the treatment of cervicogenic headache. J Manip Physiol Ther. 1995; 18:435-40.

22. Jull G, Trott P, Potter H, Zito G, Niere K, Shirley D, Emberson J, Marschner I, Richardson C. A randomized controlled trial of exercise and manipulative therapy for cervicogenic headache. Spine (Phila Pa 1976). 2002:27:1835-43

23. Haas M, Groupp E, Aickin M, Fairweather A, Ganger B, Attwood M, Cummins C, Baffes L. Dose response for chiropractic care of chronic cervicogenic headache and associated neck pain: a randomized pilot study. J Manip Physiol Ther. 2004;27:547-53.

24. Haas M, Spegman A, Peterson D, Aickin M, Vavrek D. Dose response and efficacy of spinal manipulation for chronic cervicogenic headache: a pilot randomized controlled trial. Spine J. 2010;10:117-28.

25. Speciali JG, Peres M, Bigal ME. Migraine treatment and placebo effect. Expert Rev Neurother. 2010;10:413-9.

26. de Groot FM, Voogt-Bode A, Passchier J, Berger MY, Koes BW, Verhagen AP. Headache: the placebo effects in the control groups in randomized clinical trials; an analysis of systematic reviews. J Manip Physiol Ther. 2011:34:297-305.

27. Chaibi A, Benth JS, Tuchin P, Russell MB. Chiropractic spinal manipulative therapy for migraine: a three-armed, single-blinded, placebo, randomized controlled trial. Eur J Neurol. 2017;24:143-53.

28. Finniss DG, KaptchukTJ, Miller F, Benedetti F. Biological, clinical, and ethical advances of placebo effects. Lancet. 2010;375:686-95. 
29. Chaibi A, Benth JS, Tuchin P, Russell MB. Adverse events in a chiropractic spinal manipulative therapy single-blinded, placebo, randomized controlled trial for migraineurs. Musculoskelet Sci Pract. 2017;29:66-71.
30. Mitsikostas DD, Mantonakis LI, Chalarakis NG. Nocebo is the enemy, not placebo. A meta-analysis of reported side effects after placebo treatment in headaches. Cephalalgia. 2011;31:550-61.

\section{Submit your next manuscript to BioMed Central} and we will help you at every step:

- We accept pre-submission inquiries

- Our selector tool helps you to find the most relevant journal

- We provide round the clock customer support

- Convenient online submission

- Thorough peer review

- Inclusion in PubMed and all major indexing services

- Maximum visibility for your research

Submit your manuscript at www.biomedcentral.com/submit
() Biomed Central 